\title{
Estudio de conservación del material pétreo de la Casa de Cultura del Almirante Oquendo, en San Sebastián
}

\section{Conservation study of the stone material used in the Culture House of Almirante Oquendo, in San Sebastian}

\author{
I.RODRÍGUEZ-MARIBONA*,M.ZALBIDE*,F.GARCÍAGARMILLA**,J. A.IBÁÑEZ** yS.GARÍN*** \\ * Centro Tecnológico LABEIN \\ ** Dpto. Petrología/Univ. País Vasco \\ Fecha de recepción: 19-I-99 \\ *** Teusa-Tecpropasa Euskadi, S. A. \\ Fecha de aceptación: 5-IV 99 \\ ESPAÑA
}

\section{RESUMEN}

El Patrimonio Histórico-Artístico de nuestro pais, está constituido, principalmente, por edificios de piedra de silleria, en los que las areniscas juegan un papel muy importante. Sin embargo, debido, fundamentalmente, a las condiciones medioambientales y a la contaminación, estos materiales pueden perder su matriz cementante y sufrir una desintegración. Para prevenir y frenar estos fenómenos de deterioro, se pueden emplear productos consolidantes que, en ausencia de matriz cementante, fijan los granos del material pétreo, proporcionando resistencia y durabilidad al sillar.

Para asegurar la fiabilidad de las intervenciones realizadas con estos productos, es necesario llevar a cabo unos estudios previos, al objeto de definir la idoneidad del empleo de consolidantes y seleccionar el tipo más adecuado para cada caso concreto, ya que la fiabilidad de estos productos depende, en gran medida, de la microestructura del material pétreo considerado.

El presente articulo recoge un estudio llevado a cabo sobre la arenisca de Igueldo de la fachada principal de la Casa de Cultura del Almirante Oquendo, en San Sebastián, con el fin de determinar el tratamiento de consolidación más adecuado para la alteración observada en este material pétreo.

El estudio realizado consistió, en primer lugar, en la diagnosis de las patologías presentadas en el material pétreo de este edificio. Posteriormente, se ensayaron 6 productos consolidantes comerciales sobre piedra inalterada de cantera. Por último, se validaron los resultados obtenidos, mediante ensayos en el monumento de los 3 tipos de consolidantes más adecuados, aplicados en condiciones reales de obra, eligiendo el tratamiento más idóneo para la restauración de la fachada principal del edificio.

\section{SUMMARY}

The historical heritage of our country is mainly made up of masonry, playing the sandstones an important role. However, because of the effect of environmental conditions and pollution, these materials can loose their cement, and eventually disintegrate. Chemical consolidants can be applied in order to prevent and stop these decay process, which, when there is a lack of cement, fix the stone grains, enhancing the strength and durability of the masonry.

In order to ensure the success of the application of these products in restoration works, preliminary studies should be carried out, so as to define the suitability of their use, and select the most appropriate consolidant for each specific case, as the reliability of these products is mainly determined by the microstructure of the stone material under study

This paper describes a study which was carried out on Igueldo sandstone from the main façade of the Casa de Cultura del Almirante Oquendo, in San Sebastian, which aimed at establishing the most suitable consolidation technique for the stone degradation processes observed.

Firstly, the study dealt with the diagnosis of the pathologies which affected the stone from this building. Furthermore, 6 commercial consolidants were tested on quarry stone. Finally, the results were validated applying these products in real work conditions, what enabled us to select the most appropriate treatment for the restoration of the main façade of the building 


\section{INTRODUCCIÓN}

La Casa-solar de la familia Oquendo fue construida sobre un antiguo edificio rural, en su tiempo situado tras los arenales próximos al litoral de Gros, en San Sebastián. Se reedificó en el año 1580, como casapalacio, con una estructura de muros de sillería (fachada principal) y de mampostería perimetrales y vigas y pilares de madera en su interior. La casa muestra algunos signos de la casa-palacio tipo, como es la cubierta a cuatro aguas y el alero formado por ménsulas talladas en madera, el motivo de la puerta y escudos centrales y la significación de los ventanales del piso principal. La casa se convirtió en Museo de Oquendo tras la donación del edificio en 1939, hasta que se transformó, en 1984, en Casa de Cultura de Gros (1).

La fachada principal de este edificio, orientada al Sur, es de piedra de sillería, constituida, fundamentalmente, por arenisca de Igueldo, aunque a lo largo de su historia y, especialmente, en intervenciones relativamente recientes, se han sustituido algunos sillares por otros de arenisca de Pitillas. El ambiente en el que se encuentra sometida es marítimo, muy húmedo, con un cierto grado de contaminación urbana. En la foto 1 se presenta el aspecto de la fachada principal del edificio.

El grado de alteración que presentaba la piedra arenisca de esta fachada era muy elevado, mostrando un importante nivel de arenización, con pérdida de matriz cementante. Al microscopio electrónico se puede observar la disolución de los granos de feldespato potásico del material pétreo, que, aunque se originó en etapas diagenéticas antiguas (o sea, que es original e inherente a la roca de cantera), se vio incrementada por la acción de los agentes atmosféricos, con lo que

\section{INTRODUCTION}

The House of the Oquendo family was built on an ancient rural building, which was placed, at that time, behind the sandy spot close to Gros seaboard, at San Sebastian. It was rebuilt in 1580 as a palacehouse, its structure consisting of masonry walls (main façade) and side rubblework walls, as well as internal wood beams and pillars. The house still shows some typical signs of palace-houses, as a hip roof and a chantlate of wood corbels, the ornamental works of the door and windows of the main floor, and the central coat of arms. The house was refurbished into the Museo de Oquendo after its donation in 1939. Afterwards it was transformed again into Culture House of Gros in 1984 (1).

The main façade of the building, which faces South, is a masonry wall mainly made up of Igueldo sandstone. However, during its recent history, some masonry blocks have been replaced by blocks made of Pitillas sandstone. The house is located close to the sea, in a maritime humid environment, with a certain level of pollution. Photo 1 shows the aspect of the main façade of the building.

The sandstone rock of this façade exhibited a high degree of decay, presenting an important lack of cement and detached grains. The dissolution of potasic feldspar, which took place during old diagenetical phases (thus, it being original from the quarry stone), and has been enhanced by the effect of the environmental conditions, can be appreciated through the scanning electron microscope. Some samples have completely lost their cement and

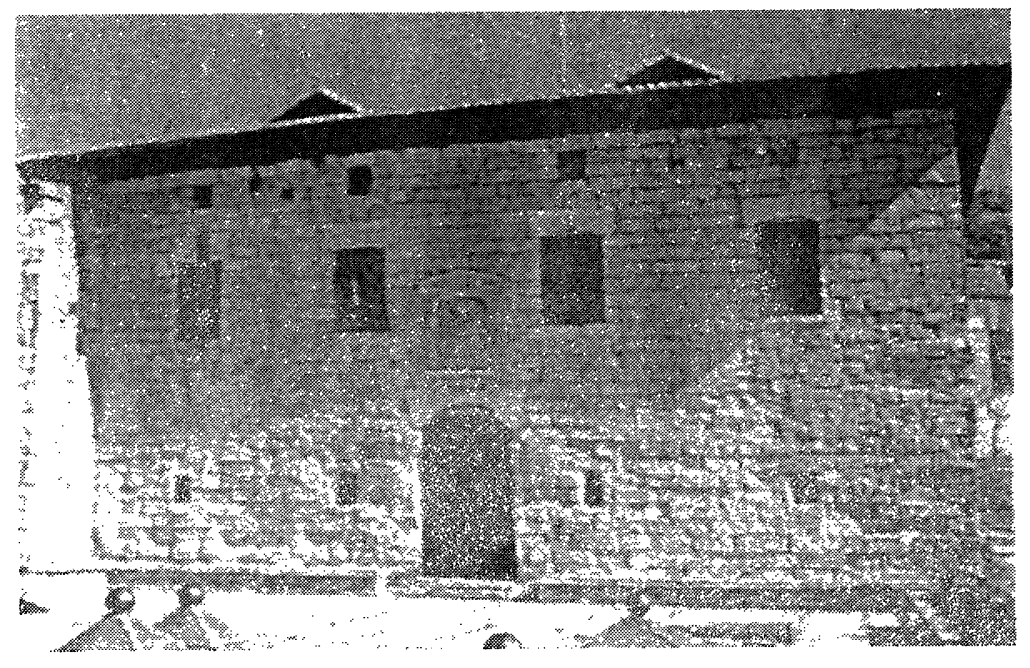

Foto 1.- Vista general de la fachada principal del edificio.

Photo 1.- General view of main front of the biaiding 
algunas muestras estaban totalmente "esponjizadas", habiendo perdido el cemento y granos de la trama originales, lo que se traduce en una baja densidad, baja coherencia y notable pérdida de peso.

Esta degradación puede ser, parcialmente, debida a la cristalización de sales en el interior del sistema poroso de la piedra, ya que se detectó una cierta cantidad de cloruros y sulfatos en su interior, provenientes del ambiente marino del edificio.

Sin embargo, el elevado grado de alteración que presentan muchos de los sillares no se debe únicamente a los factores, tales como el elevado grado de humedad y cierto nivel de contaminación por sales, sino que coexiste también la presencia de hongos, que colonizan en el interior de la piedra, creciendo a través de la matriz cementante y disgregando el material.

Debido a las patologías que presentaba la fachada principal del edificio, se decidió la restauración de la misma, según el proceso siguiente:

1. Aplicación de fungicida

2. Limpieza mediante ligero cepillado mecánico

3. Aplicación de consolidante

4. Aplicación de hidrofugante

En el presente trabajo se analiza el estudio realizado para la selección del consolidante más adecuado para este tipo de piedra y grado de alteración.

\section{MÉTODOEXPERIMENTAL}

Para la realización de este estudio, se siguieron las fases que se describen a continuación.

\section{Selección de los consolidantes a estudiar}

Los consolidantes organosilíceos que se consideraron en este estudio fueron los siguientes:

- CONSISTONE FS + CONSISTONE A, de Parrot: a base de polímeros de silicio hidrolizables y copolímeros de siloxano/acrilatos

- DURCIPIERRE, de CIMENTS LAFARGE: silicato de etilo hidrófugo

- SILEX OH, de KEIM: a base de éster de ácido salicílico, sin hidrofugante

- SM 296, de SICOF: modificación derivada del ácido silícico en solución con mezcla de disolventes alifáticos y aromáticos original grains, which has led to a low specific weight, low coherence and important weight loss.

The decay phenomena could be partially due to salt crystallisation into the internal porous system of the stone, as certain quantities of chlorides and sulphates have been detected in the internal areas of the stone.

However, the important degree of decay exhibited by many of the masonry blocks is not only due to the high degree of humidity and salt pollution, but also to the colonisation by fungi of the interior of the stone material. These organic elements grow through the cement matrix, disintegrating the material.

Due to the degree of damage exhibited by the mainfaçade of the building, the restoration of this element was undertaken. The subsequent procedure was followed:

1. Application of fungicide

2. Cleaning by means of a light manual brushing

3. Application of consolidant

4. Application of water repeller

This work analyses the study carried out for the selection of the most suitable consolidant for this type of stone and degree of decay.

\section{EXPERIMENTAL TESTING METHOD}

The study involved the tasks which are described below:

Selection of consolidants to be considered within the study

The following organosilicon consolidants were considered:

- CONSISTONE FS + CONSISTONE A, from Parrot: Based on hydrolysable silicon polymers and siloxane/ acrylate copolymers

- DURCIPIERRE, from CIMENTSLAFARGE:

hydrofugous ethyl silicate

- SILEX OH, from KEIM: based on salicylic acid ester, non hydrofugous

- SM296, from SICOF: Modification from salicylic acid and aliphatic and aromatic solvents. 
- TEGOVAKOM, de GOLDSCHMIDT: a base de éster de sílice y metilxiloxano

- WACKER OH, de WACKER SILICONE: a base de éster etílico del ácido salicílico

Con el fin de omitir los nombres comerciales de los consolidantes, así como el comportamiento de cada uno de ellos en este tipo de arenisca, se asignaron, a cada uno de estos 6 productos, números aleatorios (del $1 \mathrm{al} 6$ ), a los que se hará referencia a partir de ahora.

\section{Caracterización de la arenisca silícea de Igueldo}

La caracterización de la arenisca de Igueldo, componente de la fachada principal del edificio, se realizó mediante ensayos sobre piedra inalterada de cantera y piedra de sillería, llevando a cabo los ensayos que se describen a continuación:

*Caracterización petroquímica y microestructural: petrografía por lámina delgada,

difracción de rayos-X, microscopía electrónica de barrido

*Propiedades hídricas, realizadas según las recomendaciones RILEM (2): absorción y succión de agua

*Propiedades fisicas: densidad aparente y distribución del sistema poroso, por porosimetría de mercurio

*Durabilidad, según las recomendaciones RILEM (3): índice de desmoronamiento y resistencia a la cristalización de sales

*Propiedades mecánicas: resistencia a compresión (mediante rotura de probetas, según UNE 22185 y velocidad de propagación de ondas ultrasónicas (4))

*Análisis de sales solubles (cloruros y sulfatos)

Selección de los consolidantes más adecuados sobre piedra de cantera

Los 6 consolidantes seleccionados para el estudio se aplicaron sobre probetas de arenisca silícea de Igueldo inalterada, con el fin de analizar el comportamiento de cada uno de ellos sobre este material de cantera. El consolidante fue aplicado mediante inmersión de las probetas de piedra

Sobre los diferentes sistemas piedra de cantera/ consolidante, se realizaron los siguientes ensayos:
- TEGOVAKOM, from GOLDSCHMIDT: Based on siliceous ester and methylsiloxane

- WACKER OH, from WACKER SILICONE: Based on ethylic ester of salicylic acid.

So as not to mention the commercial names of the consolidants, as well as their corresponding performance on this type of sandstone, 6 aleatory numbers were assigned to these products (1 to 6). From now on, these consolidants will be referred to as their respective numbers.

\section{Characterisation of Igueldo's siliceous sandstone}

The characterisation of Igueldo's sandstone from the main façade of the building was carried out by several tests on quarry and masonry stone. The following tests were performed:

*Petrochemical and microstructural characterisation: thin section petrography, Xray diffraction, scanning electron microscopy

*Hydric properties, according to RILEM recommendations (2): water absorption and suction

* Physical properties: specific weight and porous system distribution, by $\mathrm{Hg}$ porosimetry

*Durability, according to RILEM Recommendations (3): slake durability test and salt crystallisation resistance

*Mechanical properties: compressive strength (destructive test, according to UNE 22185 and ultrasonic pulse (4))

*Analysis of soluble salts (chlorides and sulphates)

\section{Selection of the most suitable consolidants on quarry stone}

The selected consolidants were applied on quarry Igueldo sandstone specimens, with the purpose of analysing their performance on unweathered samples. The consolidant was applied by immersion.

The following tests were carried out on the different quarry stone/consolidant systems: 
* Caracterización microestructural, mediante microscopía electrónica de barrido

* Propiedades hídricas: absorción y succión de agua

* Propiedades físicas: densidad aparente y distribución del sistema poroso, por porosimetría de mercurio

* Durabilidad: índice de desmoronamiento y resistencia a la cristalización de sales

* Propiedades mecánicas: resistencia a compresión

* Profundidad de penetración del consolidante

* Poder hidrofugante, mediante ángulo de contacto

Validación del sistema de consolidación, sobre el monumento

Una vez seleccionados los consolidantes que mejor perecían comportarse sobre piedra de cantera, se realizó la selección final del sistema de consolidación más adecuado, mediante su aplicación en el monumento.

Como consecuencia de los resultados obtenidos en la fase anterior, se seleccionaron 3 de los consolidantes citados anteriormente. Estos productos fueron aplicados en una pequeña zona de la fachada a restaurar, empleando las técnicas de aplicación utilizadas en obra. Posteriormente, sobre pequeñas muestras de piedra consolidada extraídas del edificio, se realizaron los siguientes ensayos:

* Caracterización microestructural, mediante microscopía electrónica de barrido

* Profundidad de penetración del consolidante

Como consecuencia de esta validación final, se comprobó la viabilidad de la intervención aplicando sistemas de consolidación y se seleccionó el producto que, para este caso, resultaba ser el más idóneo.

\section{RESULTADOS OBTENIDOS}

A continuación se presentan los resultados obtenidos en los diferentes ensayos realizados:

\section{Caracterización de la arenisca de Igueldo}

Al objeto de comparar las características de la piedra inalterada de cantera y piedra degradada del monumento, se realizaron los ensayos que se describen a continuación, con el fin de valorar la degradación producida en el monumento:
* Microstructural characterisation, by scanning electron microscopy

* Hydric properties: water absorption and suction

* Physical properties: specific weight and distribution of porous system, by $\mathrm{Hg}$ porosimetry

* Durability: slake durability test and salt crystallisation resistance

* Mechanical properties: compressive strength

* Penetration depth of the consolidant

* Water repellent character, by contact angle

Validation of the most suitable consolidation system on the monument

The consolidants which, in principle, showed the best performance on quarry stone, were selected. Then, the most suitable consolidation system was selected, on the basis of its efficiency when applied to the monument.

As a consequence of the results obtained on previous tests, 3 of the above mentioned consolidants were selected. These products were applied over a small area of the main façade of the building, using real application techniques. Subsequently, the following tests were performed on small core specimens from the building:

* Microstructural characterisation, by scanning electron microscopy

* Penetration depth of the consolidant

As a result of this final validation, the reliability of the restoration works using consolidant systems was established, and the most suitable product for this specific case was selected.

\section{RESULTS}

The test results obtained are described as follows:

\section{Characterisation of Igueldo's sandstone}

With the aim of comparing the characteristics of unweathered quarry stone and damaged masonry stone, the following tests were carried out, in order to assess the degree of decay of the monument: 
* Caracterización petroquímica y microestructural. La arenisca de Igueldo es una subarcosa glauconítica, rica en cuarzo detrítico, como componente fundamental de la trama, aunque contiene otros componentes químicamente más inestables, como el feldespato potásico, las plagioclasas y los granos de glauconita que hacen que este tipo de piedra sea susceptible de sufrir disolución, lo que se traduce en una modificación de la porosidad. Son areniscas del "Flysch" terciario, pertenecientes al Eoceno, siendo su clasificación de subarcosa glauconítica (5). El tamaño de grano es elevado, lo que hace que la porosidad sea, asimismo, elevada.

* En las muestras alteradas del edificio se puede ver que, si bien la disolución de los granos de feldespato potásico ha sido, en parte, un proceso que ya sucedió en etapas diagenéticas antiguas, este proceso se ha ido incrementando con la exposición continuada a los agentes atmosféricos (fundamentalmente, humedad y contaminación salina), por lo que algunas muestras del edificio están completamente esponjizadas, con pérdida de cemento y trama originales, lo que se traduce en baja densidad, baja coherencia y notable pérdida de peso. Asimismo, se aprecian restos de colonización orgánica en forma de filamentos y esporas, que también han contribuido a propiciar un incremento de la porosidad. En la foto 2 se muestra un aspecto de esta colonización orgánica por filamentos.

* Caracterización físico-mecánica y durabilidad. En la tabla 1 se muestran los valores medios de las propiedades hídricas (absorción y succión de agua); físicas (densidad aparente y porosidad); mecánicas (resistencia a compresión) y de durabilidad (resistencia
* Petrochemical and microstructural characterisation. The Igueldo's sandstone is a glauconitic sub arkose, with a high percentage of fragmentary quartz, as main constituent of the cement. Besides, other chemically unstable compounds, such as potasic feldspar, plagioclases and glauconite grains, render a rock which can easily be dissolved, affecting its porosity. These materials are Tertiary "Flysch" sandstones, Eoceneous, which can be classified as glauconitic subarkoses (5). Their grain size is high, therefore their porosity is also high.

* The weathered samples of the building show that although the dissolution of potasic feldspar took place during old diagenetical phases, this process has been enhanced as a consequence of continuous exposure to the environmental agents (mainly moisture and salt pollution). For this reason, some areas of the building are completely weathered, exhibiting a complete lack of cement, low densities and coherence, as well as a notorious weight loss. Additionally, some fragments of organic colonisation can be appreciated as filaments and spores, which have also contributed to the increase in the porosity. Photo 2 shows a detail of this organic colonisation.

* Physico-chemical characterisation and durability. Table 1 shows the average values for hydric properties (water absorption and suction), physical (specific weight and porosity), mechanical (compressive strength) and durability (slake

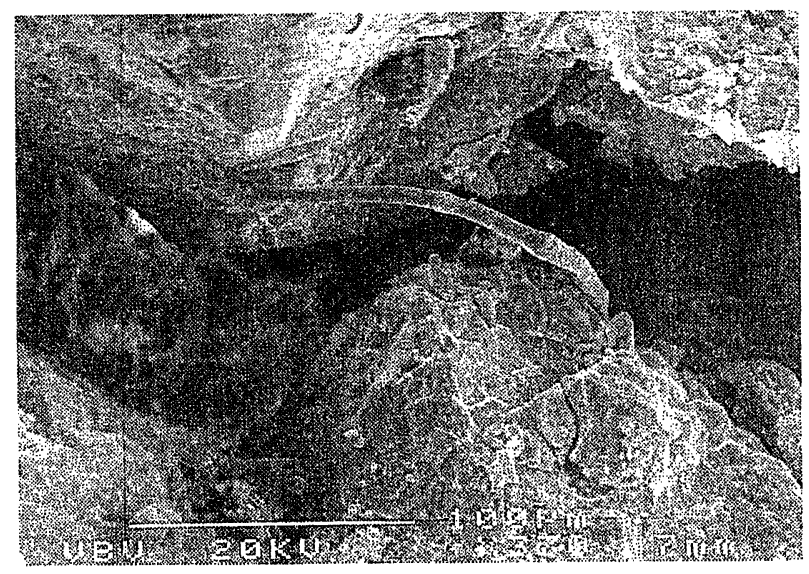

Foto 2.- Detalle de la colonización por hongos en el interior de la arenisca silícea.

Photo 2.- Fungus growth inside the monument siliceous sandstone. 
al desmoronamiento y cristalización de sales), obtenidos en piedra de cantera y del monumento, al objeto de comparar las características de la piedra inalterada y degradada, respectivamente.

De los resultados obtenidos en estos ensayos, se puede ver que, tanto la absorción como la succión de agua de este tipo de piedra son bastante elevadas, lo que facilita la degradación por disolución parcial de su cemento, tal y como se ha explicado en el apartado anterior. Este hecho es debido, principalmente, a la distribución del sistema poroso que presenta este tipo de piedra (porosidad, tamaño de poro y la curva de absorción/ desorción de mercurio), lo que facilita la entrada de agua en su interior.

La microestructura de este tipo de piedra y su alterabilidad se manifiestan en la reducida durabilidad de la misma (principalmente frente a la cristalización de sales), ya que la resistencia frente a agentes agresivos externos está condicionada, en gran medida, a la facilidad de circulación de agua por el interior del material (6).

Asimismo, sus resistencias mecánicas son bastante reducidas (5-25 $\mathrm{MPa})$. durability test and salt crystallisation). These properties have been determined on quarry and masonry stone, with the purpose of establishing a comparison between unweathered and damaged stone.

The results presented on this table show that both water absorption and suction indexes are rather high, which facilitate the partial dissolution of the cement, as described before. This fact is mainly due to the distribution of the porous system of this type of stone (porosity, pore size and $\mathrm{Hg}$ absorption/desorption curve), thus enhancing the water penetration through the material.

The microstructure of this type of stone and its weatherability are reflected on its poor durability (against salt crystallisation), as this property is closely related to the flow of water through the material (6).

Besides, its mechanical strength is rather low (5-25 MPa).

TABLA $1-$ TABLE 1

CARACTERIZACIÓN FÍSICO-MECÁNICA Y DURABILIDAD EN PIEDRA DE CANTERA Y SILLERÍA (PHYSICO-CHEMICAL CHARACTERISATION AND DURABILITY OF QUARRY AND MASONRY STONE)

\begin{tabular}{|c|c|c|c|c|}
\hline $\begin{array}{l}\text { PROPIEDAD } \\
\text { (PROPERTY) }\end{array}$ & \multicolumn{2}{|c|}{$\begin{array}{l}\text { CARACTERÍSTICA } \\
\text { (CHARACTERISTIC) }\end{array}$} & $\begin{array}{l}\text { PROCEDENCIA } \\
\text { DE PIEDRA } \\
\text { (STONE SOURCE) }\end{array}$ & $\begin{array}{l}\text { RESULTADO } \\
\text { (RESULT) }\end{array}$ \\
\hline \multirow{4}{*}{$\begin{array}{l}\text { HÍDRICAS } \\
\text { (HYDRIC) }\end{array}$} & \multirow{2}{*}{\multicolumn{2}{|c|}{$\begin{array}{l}\text { Absorción de agua (\%) } \\
\text { (Water absorption) }\end{array}$}} & Cantera/Quarry & 4,97 \\
\hline & & & Silleria/Masonry & 5,35 \\
\hline & \multirow{2}{*}{\multicolumn{2}{|c|}{$\begin{array}{c}\text { Succión capilar }\left(\mathrm{g} / \mathrm{cm}^{2} / \mathrm{min}\right) \\
\text { (Water suction) }\end{array}$}} & Cantera/Quarry & 0,063 \\
\hline & & & SilleríaMasonry & 0,092 \\
\hline \multirow{6}{*}{$\begin{array}{l}\text { FÍSICAS } \\
\text { (PHYSICAL) }\end{array}$} & \multirow{2}{*}{\multicolumn{2}{|c|}{$\begin{array}{c}\text { Densidad aparente }\left(\mathrm{Kg} / \mathrm{m}^{3}\right) \\
\text { (Specific weight) }\end{array}$}} & Cantera/Quarry & 2185 \\
\hline & & & Sillería/Masonry & 2144 \\
\hline & \multirow{4}{*}{$\begin{array}{l}\text { Porosidad } \\
\text { (Porosity) }\end{array}$} & \multirow{2}{*}{$\begin{array}{l}\text { Porosidad (\%) } \\
\text { (Porosity) }\end{array}$} & Cantera'Quarry & 18,29 \\
\hline & & & SilleríaMasonry & 23,42 \\
\hline & & \multirow{2}{*}{$\begin{array}{l}\text { Diám. poro }(\mathrm{A}) \\
\text { (Pore diameter) }\end{array}$} & Cantera/Quarry & $3 \times 10^{4}$ \\
\hline & & & Silleria/Masonry & $2 \times 10^{5}$ \\
\hline \multirow{4}{*}{$\begin{array}{l}\text { DURABILIDAD } \\
\text { (DURABILITY) }\end{array}$} & \multirow{2}{*}{\multicolumn{2}{|c|}{$\begin{array}{l}\text { Índice desmoronamiento } \\
\text { (Slake durability test) }\end{array}$}} & Cantera/Quarry & 94,68 \\
\hline & & & Silleria/Masonry & 96,36 \\
\hline & \multirow{2}{*}{\multicolumn{2}{|c|}{$\begin{array}{l}\text { Cristal. sales. Pérdida peso (\%) } \\
\text { (Salt cristal. Weight loss) }\end{array}$}} & Cantera/Quarry & 82,26 \\
\hline & & & Silleria/Masonry & 80,41 \\
\hline \multirow{2}{*}{$\begin{array}{l}\text { MECÁNICAS } \\
\text { (MECHANICAL) }\end{array}$} & \multirow{2}{*}{\multicolumn{2}{|c|}{$\begin{array}{l}\text { Resist. a compresión (MPa) } \\
\text { (Compressive Strength) }\end{array}$}} & Cantera/Quarry & 24,15 \\
\hline & & & SilleriaMasonry & 13,38 \\
\hline
\end{tabular}


Como se ha comentado anteriormente, la piedra del monumento se encontraba muy degradada, como así se puede constatar por los valores obtenidos en los ensayos realizados, en los que la pérdida parcial de cemento por disolución del mismo se manifiesta en una porosidad mayor y, por tanto, menor densidad, así como en una facilidad de circulación de agua por el interior de la piedra que resulta superior al caso de la roca inalterada de cantera, lo que disminuye, asimismo, la durabilidad del material.

* Análisis de sales solubles. En la tabla 2 se presenta el análisis de las sales solubles (cloruros y sulfatos) obtenidas en diferentes puntos del interior del material pétreo del monumento.

Como puede observarse en esta tabla, la cristalización de cloruros -y sobre todo de sulfatos- en el interior de la piedra arenisca, ha podido contribuir a la alteración que se observa; aunque la incidencia de otros factores, tales como la disolución parcial del cemento del material por la circulación de agua y la presencia de organismos biológicos, han podido contribuir también, en gran medida, a este deterioro.

\section{Selección del consolidante más adecuado}

Ensayos comparativos realizados sobre arenisca silícea de Oquendo de cantera y monumental (7), indican que el comportamiento de los diferentes consolidantes sobre piedra de cantera y sillería es muy similar, por lo que parece que el estado de degradación de la piedra no resulta muy influyente en este sentido. Por ello, al objeto de alterar lo mínimo posible el monumento, parte de los ensayos se realizaron sobre piedra de cantera.

De todos los ensayos que se exponen en el apartado 2, realizados en piedra de cantera, sobre la que se aplicaron los diferentes consolidantes seleccionados, se exponen los que han resultado ser los más discriminatorios a la hora de elegir el producto de tratamiento que mejor se adaptaba a este tipo de piedra.
As above mentioned, the masonry stone exhibited a high degree of decay, as shown by the tests results. The partial loss of cement by dissolution results in a higher porosity, and, therefore, a lower density, thus facilitating a greater ingress of water into the masonry stone as compared to the quarry stone. rendering a non durable material.

* Analysis of soluble salts. Table 2 shows the analysis of soluble salts (chlorides and sulphates) obtained for different areas of the masonry stone of the monument.

As shown on table, the crystallisation of chlorides, and, above all, sulphates, inside the sandstone, could have, in certain way, contributed to the existing degree of decay, although the influence of factors such as the partial dissolution of cement due to the flow of water, as well as the growth of organisms, could have also contributed to the decay processes.

\section{Selection of the most suitable consolidant}

Comparative tests carried out on quarry and masonry Oquendo's sandstone (7), showed that the performance of the various consolidants selected on both types of stone was very similar. Therefore, the degree of decay condition of the stone did not affect their behaviour significantly. For this reason, and in order to minimise the damage to the monument, some tests were carried out on quarry stone.

Of all the tests which were carried out on differently consolidated quarry stone, only those which resulted more discriminatory when selecting the most suitable consolidation treatment for this type of stone will be described. These tests were: water

TABLA 2 - TABLE 2

ANÁLISIS DE SALES SOLUBLES DE LA ARENISCA DE LA CASA DE OQUENDO (ANALYSIS OF SOLUBLE SALTS OF OQUENDO'S HOUSE SANDSTONE)

\begin{tabular}{|c|c|c|c|c|}
\hline \multirow{2}{*}{$\begin{array}{c}\text { MUESTRA } \\
\text { (SAMPLE) }\end{array}$} & \multicolumn{2}{|c|}{$\begin{array}{c}\text { CLORUROS }(\mathrm{Cl})(\%) \\
\text { (CHLORIDES) }\end{array}$} & \multicolumn{2}{c|}{$\begin{array}{c}\left.\text { SULFATOS (SO }{ }^{3}\right)(\%) \\
\text { (SULPHATES) }\end{array}$} \\
\cline { 2 - 5 } & $\begin{array}{c}\text { SUPERFICIE } \\
\text { (SURFACE) }\end{array}$ & $\begin{array}{c}\text { INTERIOR } \\
\text { (INTERIOR) }\end{array}$ & $\begin{array}{c}\text { SUPERFICIE } \\
\text { (SURFACE) }\end{array}$ & $\begin{array}{c}\text { INTERIOR } \\
\text { (INTERIOR) }\end{array}$ \\
\hline 1 & 0,35 & 0,20 & 0,18 & 0,03 \\
\hline 2 & 0,20 & 0,01 & 0,69 & $<0,01$ \\
\hline 3 & 0,14 & 0,22 & 0,82 & $<0,01$ \\
\hline 4 & 0,02 & 0,07 & 0,78 & 0,02 \\
\hline 5 & 0,11 & 0,05 & 1,03 & 0,01 \\
\hline
\end{tabular}


Estos ensayos han sido: absorción (Figura 1), succión de agua (Figura 2) y resistencia a la cristalización de sales (Figura 3 ).

Como se puede observar en las gráficas anteriores, así como en el resto de los ensayos realizados, los consolidantes 2,5 y 6 eran los que mejores propiedades presentaban en este tipo de arenisca. Por este motivo, se procedió a la aplicación de estos consolidantes en el monumento, al objeto de verificar su buen comportamiento, así como de determinar la profundidad de penetración de ambos, propiedad fundamental para asegurar la correcta consolidación del edificio. Las profundidades medias de penetración de estos productos fueron de $36,15 \mathrm{~mm}$ para el $\mathrm{N}^{\circ} 5 ; 9,43 \mathrm{~mm}$ para el $\mathrm{N}^{\circ} 2$ y $4,52 \mathrm{~mm}$ para el $\mathrm{N}^{\circ} 6$. Dada la escasa profundidad de penetración de este último, se descartó su empleo para este tipo de piedra, continuando el estudio únicamente con los números 2 y 5 .

Por otra parte, se realizaron una serie de observaciones al microscopio electrónico de barrido, al objeto de determinar la deposición de ambos consolidantes en el interior del sistema poroso de la piedra. En las fotografias 3 y 4 se observa el aspecto del consolidante $N^{\circ} 5$ y 2 , respectivamente, una vez que la piedra del monumento se había impregnado con ellos. Como se puede observar en estas ultrafotografias, parece que, aunque no siempre era fácil observar la presencia del tratamiento recubriendo los granos de la roca, el consolidante $\mathrm{N}^{\circ} 5$ aparece como una pasta más compacta que recubre los granos, ajustándose mejor a los bordes originales de los granos detríticos. Por el contrario, el $\mathrm{N}^{\circ} 2$ tiene una estructuración, una vez solidificado, mucho más porosa, que no se ciñe correctamente a los bordes de grano de la trama, a la vez que más heterogénea, formando grandes aglomeraciones en algunos puntos.

Dado que, por una parte, el comportamiento del consolidante $\mathrm{N}^{\circ} 5$ parecía ser, en principio, ligeramente mejor que el $\mathrm{N}^{0} 2$, y puesto que, por otra, su profundidad de penetración era mayor, se decidió consolidar el edificio con el primero.

Las características del producto de consolidación con el que se realizó el tratamiento superficial de la fachada fueron:

- Propiedades: efecto consolidante e hidrofugante

- Naturaleza: silicato de etilo asociado a catalizador neutro y resina de metilo

- Acción: formación de gel silíceo (consolidación) y resina de polisiloxano (hidrofugación). Líquido densidad $0,9 \mathrm{~kg} / 1$ absorption (Figure 1), water suction (Figure 2) and salt crystallisation resistance (Figure 3).

As shown on previous figures, as well as on the rest of the tests carried out, consolidants 2, 5 and 6 conferred the best properties to this type of sandstone. With the aim of validating the behaviour of these 3 products in real work conditions, and determining their penetration depth (essential property to ensure a correct consolidation of the building), these consolidants were applied on the monument. The average penetration depths were 36,15 mm, for $\mathrm{Nr}$. 5; 9,43 $\mathrm{mm}$ for $\mathrm{Nr} .2$ and 4,52 $\mathrm{mm}$ for Nr. 6. On the basis of the low penetration depth achieved with the last product, its application on this type of stone was rejected. Therefore, the study was continued with both $\mathrm{Nr} .2$ and 5 products.

On the other hand, scanning electron microscope tests were carried out in order to determine the deposition of both consolidants into the porous system of the stone. Photos 3 and 4 show the appearance of consolidants $\mathrm{Nr} .5$ and 2, respectively, once the stone material had been treated with them. Although the observation of the consolidant covering the stone grains was not easy, consolidant Nr. 5 appeared as a compact layer, completely fitting the original edge of the detritic grains. On the contrary, once the consolidant Nr. 2 had solidified, its structure became much more porous, and did not adjust well to the grain edges. Besides, the structure of this last product was much more heterogeneous, presenting large agglutinations in some areas.

Provided that, on one hand, the performance of consolidant Nr. 5 seemed better than $\mathrm{Nr}$. 2, and, on the other hand, its penetration depth was higher, the main façade of the building was consolidated with this product.

The main characteristics of the consolidant used for the surface treatment of the façade were:

-Properties: consolidant and water repellent effect

-Nature: ethyl silicate associated to neutral catalyst and methyl resin

- Action: formation of silica gel (consolidation) and polisiloxane resin (water repeller). Liquid: density $0,9 \mathrm{~kg} / \mathrm{l}$ 


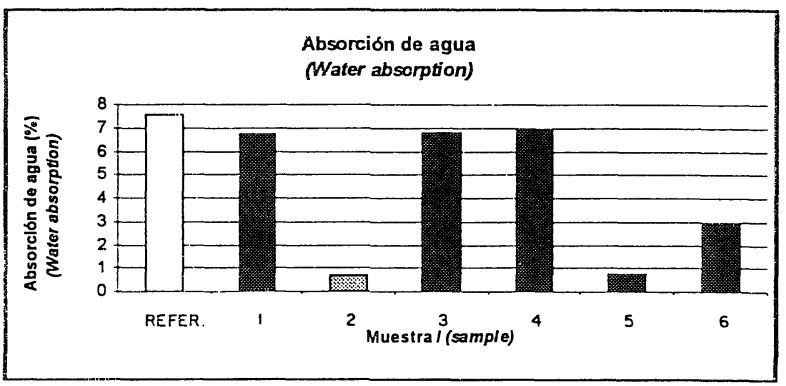

Figura 1.- Absorción de agua de la arenisca inalterada de Igueldo consolidada.

Figure 1.- Water absorption of consolidated unweathered Igueldo's sandstone.

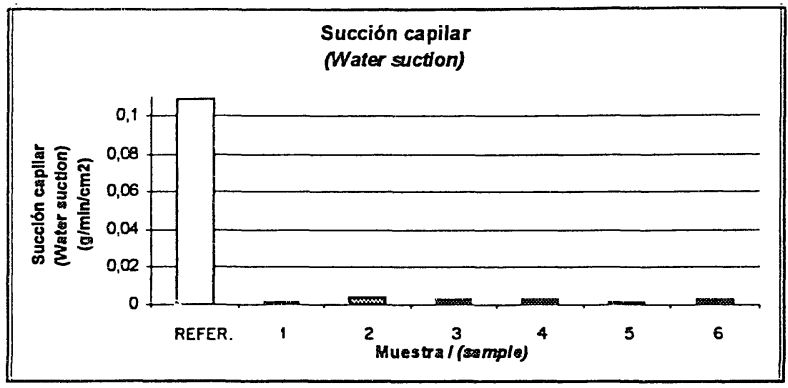

Figura 2.- Succión capilar de la arenisca inalterada de Igueldo consolidada.

Figure 2.- Water suction of consolidated unweathered Igueldo's sandstone.



Figura 3.- Resistencia a la cristalización de sales de la arenisca de Igueldo consolidada.

Figure 3. Salt crystallisation resistance of consolidated Igueldo's sandstone.

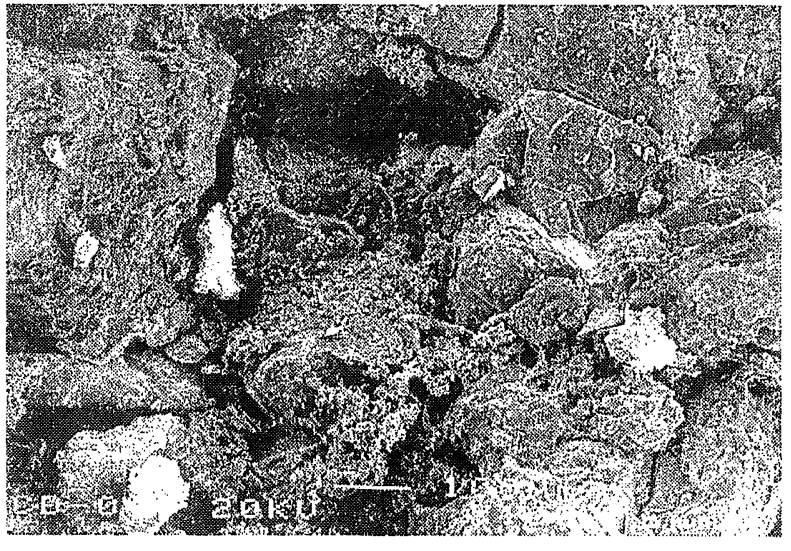

Foto 3.- Recubrimiento de los granos de sílice por el consolidante $\mathrm{N}^{\circ} 5$.

Photo 3.- Coating of silica grains by conso: dant Nr. 5.

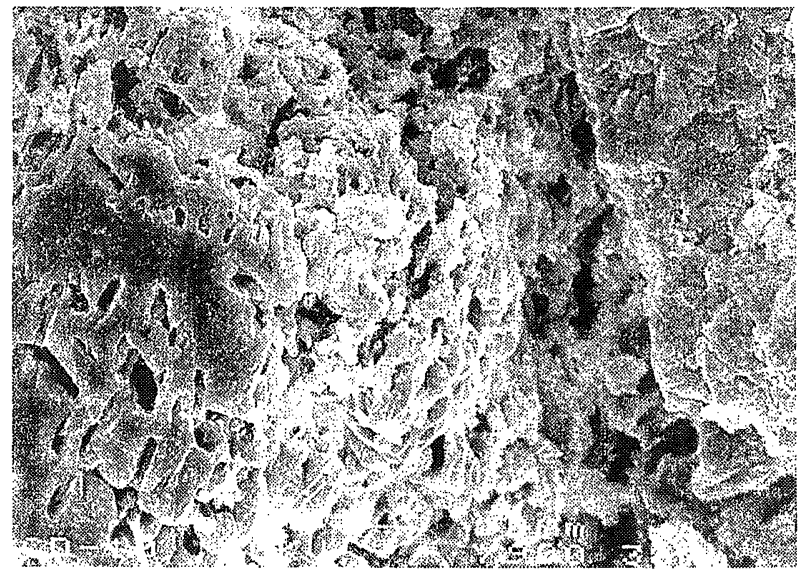

Foto 4.- Recubrimiento de los granos de silice por el consolidante $\mathrm{N}^{\circ} 2$.

Photo 4.- Coating of silica grains by consolidant $\mathrm{Nr} .2$. 


\section{CONCLUSIONES}

A la vista de los resultados obtenidos en la experimentación, pueden extraerse las siguientes conclusiones:

1. La arenisca de Igueldo es una piedra fácilmente meteorizable, dado su sistema poroso, que facilita la circulación de agua en su interior, lo que, a su vez, facilita los procesos de disolución del cemento de la roca y la cristalización de sales en su interior, procesos que, unidos a la colonización por agentes biológicos de la arenisca de la Casa de Oquendo, han provocado el avanzado estado de deterioro de los sillares de arenisca de la fachada principal del edificio.

2. El grave deterioro de los sillares, con importantes pérdidas de sección, arenización de la piedra, incoherencia y pérdida de cemento, hacía necesaria la consolidación y posterior hidrofugación de esta fachada.

3. La caracterización realizada sobre piedra inalterada de cantera y piedra degradada del monumento denota el aumento de porosidad y, por tanto, de las propiedades hídricas y disminución de durabilidad y resistencias mecánicas sufridas por la piedra de sillería del monumento.

4. Los valores de las propiedades físico-mecánicas, hídricas y de durabilidad obtenidos sobre la piedra de cantera consolidada con los 6 productos seleccionados permitió elegir 3 de ellos como los más indicados para este tipo de piedra. Los ensayos de absorción y succión de agua, así como el de cristalización de sales han resultado ser los más concluyentes para la selección del tipo de producto de consolidación más indicado.

5. La aplicación in situ (en una pequeña zona) de los 3 consolidantes considerados, y la selección de uno de ellos en función de la profundidad de penetración del mismo y de la impregnación de los granos de sílice de la arenisca, observada mediante microscopía electrónica de barrido, permitió validar los ensayos realizados sobre piedra inalterada de cantera.

6. Como conclusión final, confirmar que, para la selección de los tratamientos superficiales más adecuados, según el tipo de piedra y edificio, es necesario realizar un estudio profundo e individualizado para cada caso concreto, ya que no existe una solución universal que pueda ser aplicada en todos los casos.

\section{AGRADECIMIENTOS}

Este trabajo ha sido realizado mediante subvenciones ATYCA-MINER y Universidad-Empresa (Gobierno

\section{CONCLUSIONS}

The following conclusions can be drawn from the results of the testing strategy followed:

1. Igueldo's sandstone is a highly weatherable stone, mainly due to its porous system, which facilitates the flow of water through the material, enhancing the dissolution of cement and salt crystallisation processes. These phenomena, jointly with the growth of biological organisms inside the Oquendo's sandstone, had lead to the high degree of decay observed at the sandstone of the main façade.

2. Consolidation and subsequent hydrofugation of this façade were crucial due to the serious degree of decay of the masonry, with important section losses, unbonding of grains and loss of cement.

3. The characterisation of the unweathered quarry and damaged masonry stone showed a higher porosity for the masonry material, i.e. significant hydric properties, and low durability and mechanical properties.

4. The values of physico-mechanical, hydric and durability properties for the consolidated quarry stone allowed the selection of 3 out of the 6 preselected products, as most suitable, in principle, for, this type of stone. Absorption, suction and salt crystallisation tests have proved as those most effective for the assessment of consolidation products.

5. The in situ application of the selected consolidants, as well as the penetration depth and morphology of grain impregnation, as observed through scanning electron microscopy, permitted the validation of the tests carried out on quarry stone.

6. Finally, it can be concluded that, for the selection of the most suitable surface treatment of each specific type of stone and building, a detailed and tailored study is required, as there is not any global solution to be applied in all cases.

\section{ACKNOWLEDGEMENTS}

This work has been funded by the ATYCA-MINER and Universidad-Empresa programmes. The project work 
Vasco), por lo que se agradece a ambos organismos su contribución para la realización de este proyecto. team wants to acknowledge both the Spanish Government and the Basque Government for their contribution to the development of this project.

\section{BIBLIOGRAFÍA}

(1) ITXASOA: Tomo V, Ed. Etor, San Sebastián, (1984), pp. 84-86

(2) RILEM COMISSION 25-PEM. "Protection et érosion des monuments”, Ed. Bordas-Dunod, Paris (1978)

(3) RILEM: "Essais recommandées pour mesurer l'altération des pierres et évaluer l'éfficacité des méthodes de traitement", Matériaux et Constructions, 13,(75), (1980)

(4) MONTOTO, M., CALLEJA, L., PÉREZ, B., ESBERT, R.M.: "Evaluation in situ of the state of deterioration of monumental stones by non-destructive ultrasonic techniques", Material Issues in Art and Archaeology II, MRS. (1991), pp. 273-284

(5) PETTIJOHN, F.J., POTTER, P.PE, SIEVER, R.: "Sand and Sandstone", Springer-Verlag, New York, (1987), p. 553

(6) ESBERT, R.M., ORDAZ, J., MARCOS, R.M., VALDEÓN, L.: "Propiedades físicas a considerar en el deterioro de las rocas como materiales de construcción”, Rev. de Inv. Geológicas, Vol. 44-45, (1987-88), pp. 303-311

(7) RODRÍGUEZ-MARIBONA, I.: “Estudio sobre los consolidantes a aplicar en areniscas silícea de la C.A.P.V.", Bilbao, (no publicado) (1998)

\section{Publicación del Instituto Eduardo Torroja - CSIC}

\section{Número monográfico de INFORMES}

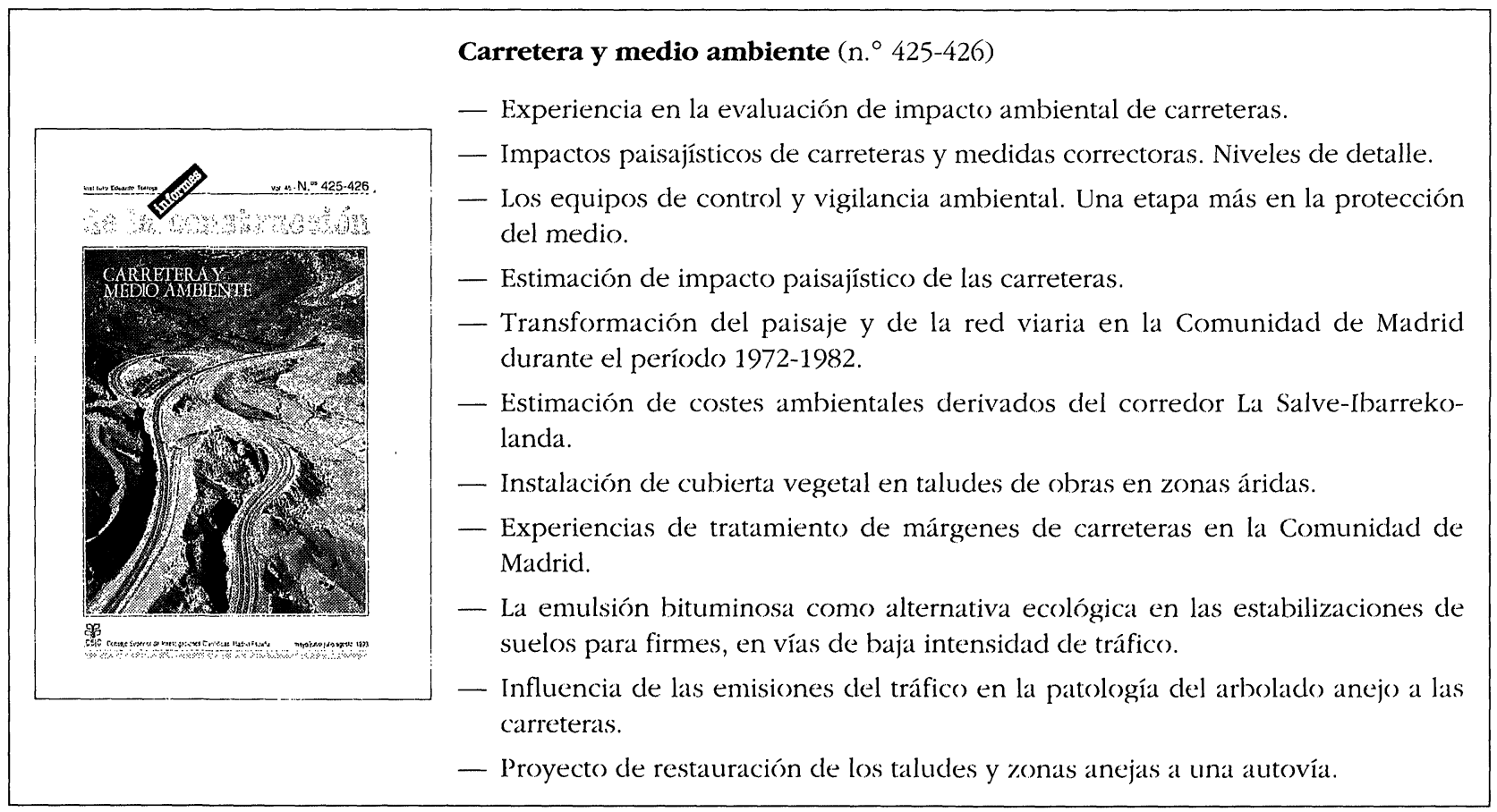

